\title{
Persistent Abdominal Pain as Rare Complication of Duodenal Perforation From an Inferior Vena Cava Filter
}

\author{
Walid Khan ${ }^{1}$, Wei Zhang ${ }^{2}$, Virginia Clark ${ }^{2}$ \\ 1. Department of Internal Medicine, University of Florida, Gainesville, USA 2. Department of Gastroenterology and \\ Hepatology, University of Florida, Gainesville, USA
}

Corresponding author: Walid Khan,wkhan8827@gmail.com

\begin{abstract}
Deep vein thrombosis (DVT) continues to be a significant source of morbidity for surgical patients. Inferior vena cava (IVC) filter placement is indicated for DVT in patients who have contraindications to anticoagulation or anticoagulation failure. Over the last decade, there is an exponential increase in IVC filter placement with increased complications reported. These include IVC penetration, IVC occlusion, insertion complication and filter migration. We report a rare case of symptomatic duodenal perforation by an IVC filter migration. This case illustrates that even though IVC migration and perforation is a rare complication, it should be recognized as a potential cause for gastrointestinal (GI) symptoms in these patients.
\end{abstract}

Categories: Cardiac/Thoracic/Vascular Surgery, Radiology, Gastroenterology

Keywords: ivc filter, duodenum, perforation, chronic abdominal pain

\section{Introduction}

Inferior vena cava (IVC) filters came to be widely used in the 1970s for the prevention of pulmonary emboli. The only accepted indication for placement has been patients with venous thromboembolism (VTE) who have contraindications to anticoagulation, failure of anticoagulation, or complications to the anticoagulation they were on previously. However, with increased ease and the relative safety of the procedure, more filters are being used for patients that are considered high risk for thromboembolism especially after trauma [1]. From 1998 to 2005, it was seen that the rate of placement for prophylactic measures increased by $157 \%$ [2]. Despite it being such a common procedure, studies have shown that IVC filters do not have a statistically significant effect in reducing pulmonary embolism rates [3]. However, the filters have significant risks with studies show that patients had a higher incidence rate of deep vein thrombosis (DVT) after receiving an IVC filter since the underlying predisposition to thrombosis does not lessen with the placement of a filter [4]. One of the rare, later complications of IVC filter placement is migration into surrounding structures including the duodenum which can present with abdominal pain, gastrointestinal (GI) bleeding, or other nonspecific GI symptoms [5]. We present a patient with recurrent

Review began $11 / 25 / 2020$ Review ended 02/05/2021 Published 02/06/2021

\section{() Copyright 2021}

Khan et al. This is an open access article distributed under the terms of the Creative Commons Attribution License CC-BY 4.0., which permits unrestricted use, distribution, and reproduction in any medium, provided the original author and source are credited. abdominal pain following IVC migration into the duodenum.

The case was previously presented at ACG Annual Meeting 2019 in San Antonio, Texas

\section{Case Presentation}

A 33-year-old woman with a history of T11 paraplegia secondary to a motor vehicle collision, recurrent lower extremity thrombi on warfarin, and placement of a Cook Celect IVC filter in 2008 by vascular surgery as a prophylactic measure following neurosurgical intervention after MVC presented to the emergency department for the 10th time in a one-year period for acute worsening of chronic epigastric pain. She described the pain as an intermittent sharp pain that was more frequent at nighttime while lying down with no provoking or alleviating factors. Review of systems was negative outside the abdominal pain with patient denying any diarrhea, hematochezia, hematemesis, nausea, vomiting. On examination, there was tenderness to palpation in the epigastric and right upper quadrant of her abdomen with mild guarding with no distension or rigidity noted. Labs including complete blood count and basic metabolic panel were unremarkable. Computed tomography (CT) scan showed a longstanding IVC occlusion with multiple collaterals and displaced IVC filter into duodenum and into the L3 vertebral body (Figures 1,2). 


\section{Cureus}

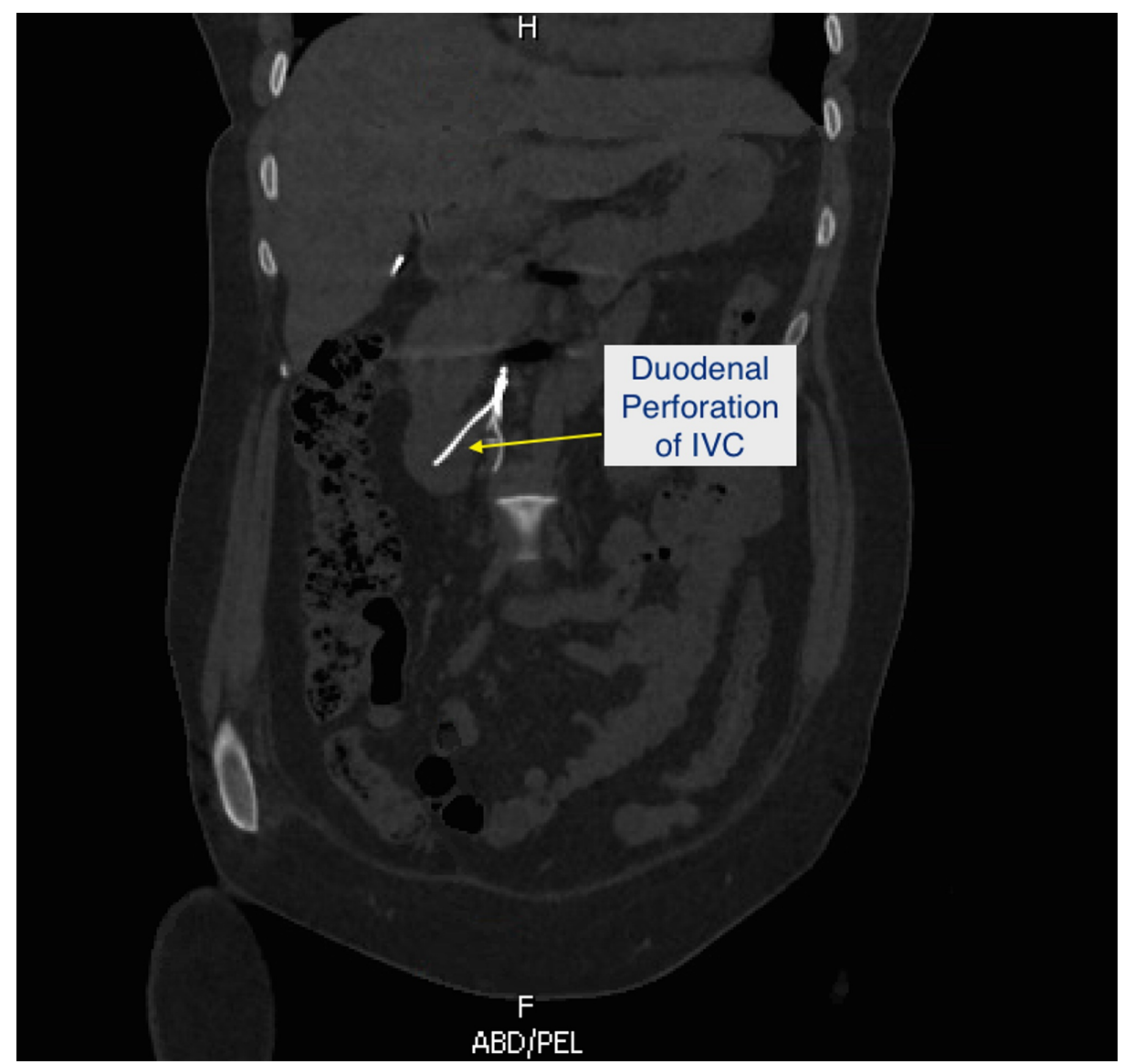

FIGURE 1: CT scan showing IVC filter penetration into duodenum. CT: computed tomography; IVC: inferior vena cava. 


\section{Cureus}

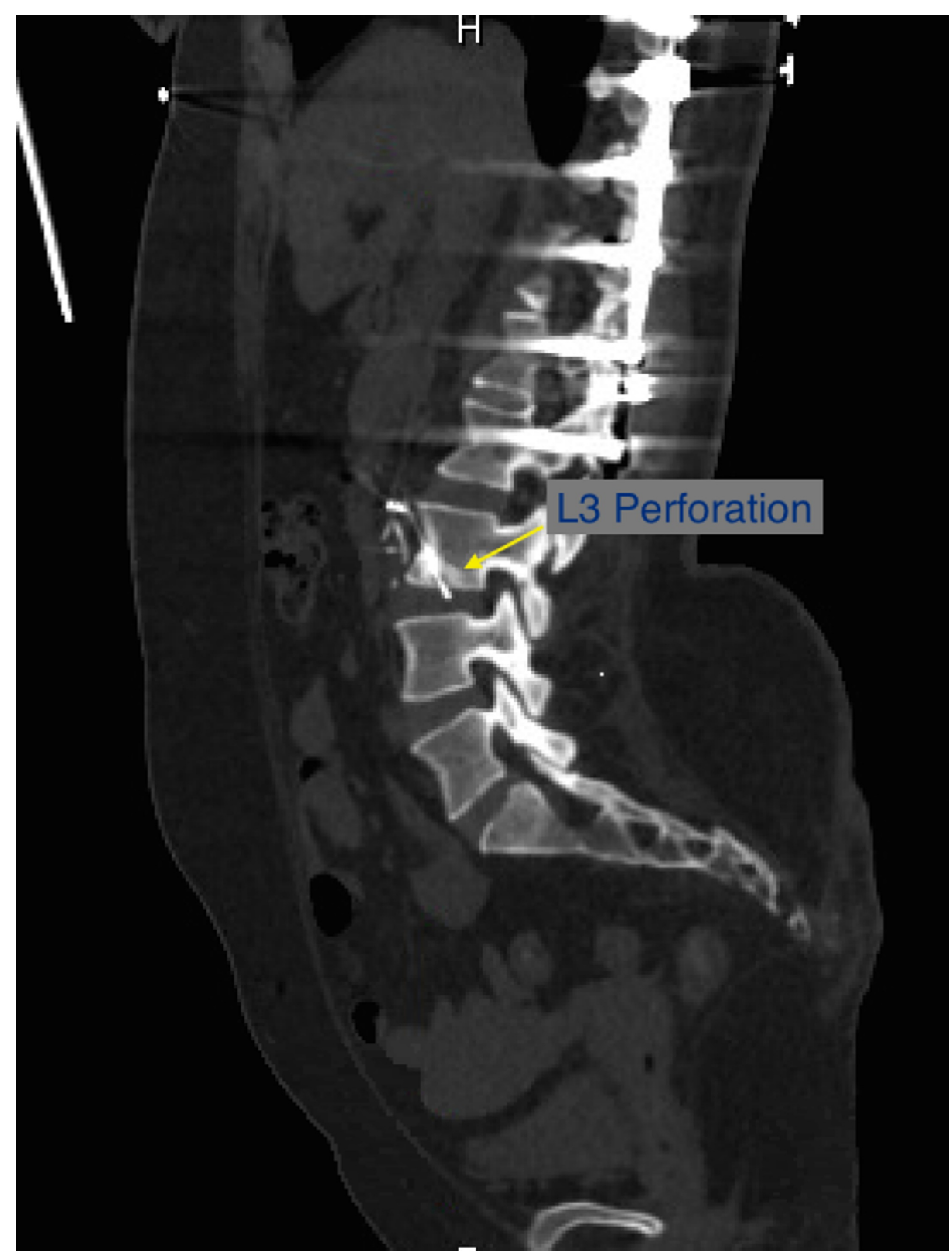

FIGURE 2: IVC filter penetrating into L3 vertebra.

IVC: inferior vena cava.

An esophagogastroduodenoscopy (EGD) was done showing prong migration to the second portion of the duodenum (Figure 3). This was distal to the ampulla and penetrating the duodenal wall with the tip embedding the opposite wall. There was no ulceration or bleeding at the site of penetration. Rat tooth forceps were used to displace the tip that was embedded in the opposite wall 


\section{Cureus}

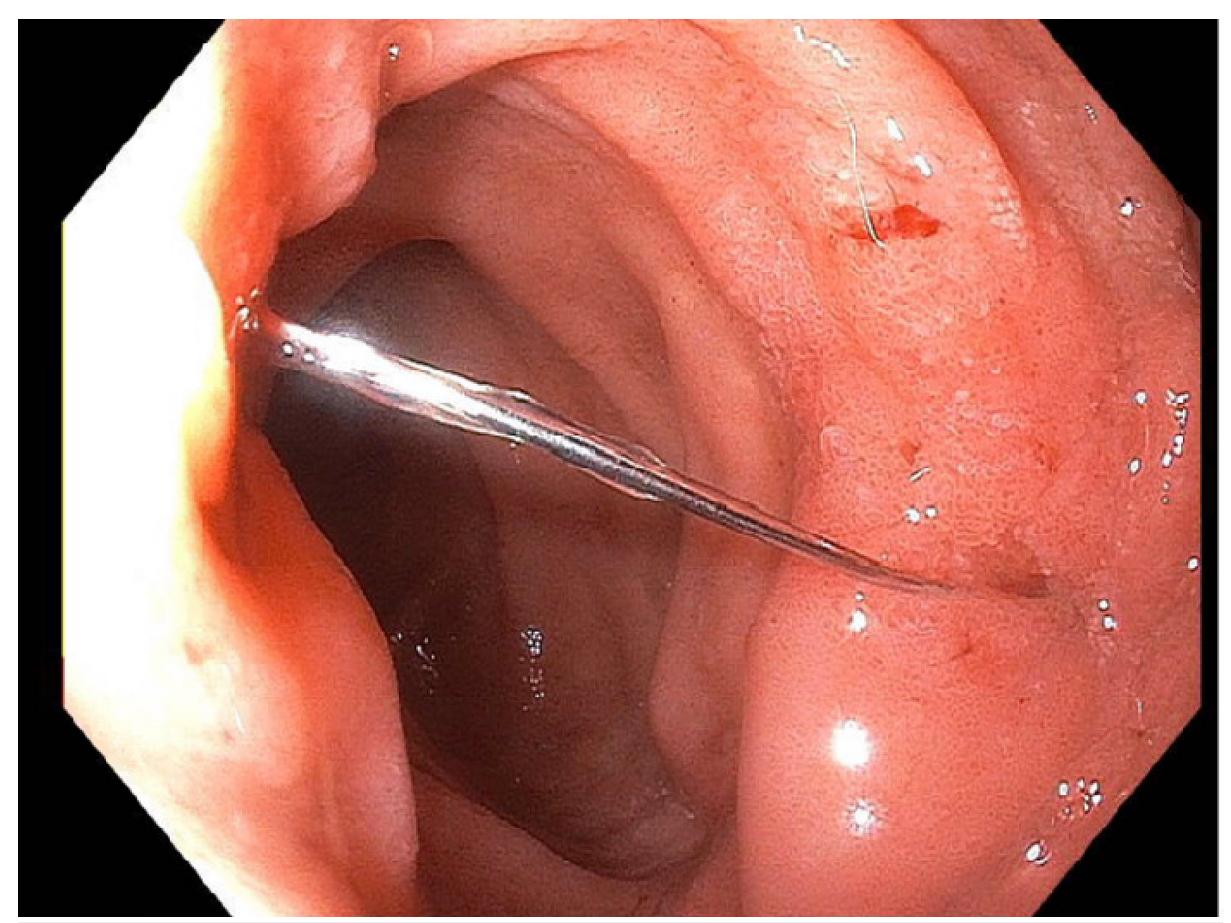

FIGURE 3: IVC strut penetrating duodenum.

IVC: inferior vena cava.

Due to multiple workups otherwise being negative for her abdominal pain with EGD and imaging findings consistent with duodenal perforation, vascular surgery was consulted and an elective surgery with open IVC filter extraction with duodenal and caval repair was scheduled.

\section{Discussion}

IVC filters were first approved in the 1970s by the Food and Drug Administration (FDA) to decrease the risk of pulmonary emboli in patients where anticoagulation was not able to be used due to either contraindications or ineffectiveness. With off-label indications for placement such as prophylaxis in trauma patients and increased ease of placement, the frequency has increased. With this, an increased prevalence of complications has also been noted [1]. This increased observed prevalence of complications could partly be due to the ease of obtaining CT scans or EGD in present times allowing easier diagnosis. These complications also increase in frequency with the time that the filters have been in place so it is likely that these filters were not in place long enough for these complications to be noted [6]. IVC filters are meant to be a temporary measure with the FDA recommending that they be removed within 25-54 days. Due to a variety of factors, retrieval is often not even attempted with one large study estimating the overall retrieval rate to be around 34\% despite a success rate of $80 \%-90 \%$ [7]. Much of this is due to loss of follow-up, but additional risk factors were mostly related to life expectancy or the root cause such as DVT, bleeding, or PE being unresolved [8]. One effective method of ensuring removal has been increasing physician education and patient programs ensuring regular follow-up; with these methods, $75 \%$ of temporary filters are removed [9].

Potential complications of IVC filter placement include DVTs, IVC thrombosis, IVC migration, perforation of either arterial or venous vessels, or perforation of surrounding structures [10]. Perforation has been reported to include structures such as the duodenum, aorta, portal veins, diaphragm, large intestine, and retroperitoneum. A systematic review by Jia et al. showed 9002 patients with IVC filters in cited studies with 322 cases of organ penetration with the duodenum being the most commonly involved organ (123 cases). Only $8 \%$ of the total 322 cases were noted to be symptomatic [11]. Because symptomatic duodenal perforation is a rare complication, there are no guidelines regarding diagnostic or treatment strategies. However, all current case reports on the topic of duodenal perforation have shown that the filters were placed more than 10 months before the onset of symptoms [6]. This further emphasizes the importance of a system to ensure timely removal of IVC filters such as education for physicians or automatically scheduled follow-up. Another factor that appears to play a role in IVC filter complications is the geometry and brand of the filter placed. Our patient received a Celect filter which has a conical shape that is more commonly associated with a high rate of penetration versus other types of IVC filters [11-13]. However certain brands of conical filters seem to have a much lower rate of penetration associated with their use when compared with the Celect filter [14]. Standardized studies need to be done comparing different shapes and brands to 
further analyze the role of these factors. The Celect filter is a temporary filter and this just further stresses the importance of staying vigilant in regards to removing these temporary IVC filters when possible.

The two methods of removal in the setting of perforation are open and endovascular repair. A systematic review article by Malgor and Labropulos showed in the setting of symptomatic duodenal perforation by IVC filters, 20/21 filters were removed by open surgery. 19/20 of these filters were placed between 7 and 180 months before patient presentation showing the prolonged time course between placement and symptoms secondary to perforation [6]. In the systematic review by Jia et al. describing all complications requiring removal, 4/83 IVC filters were removed using endovascular retrieval [11]. However, endovascular retrieval is increasingly being recognized as a safe option saving the patient from an invasive open repair in selective patients [15-17].

\section{Conclusions}

This case report emphasizes the importance of keeping IVC filter migration and penetration on the differential for a myriad of GI symptoms. Our patient had her temporary Celect filter in place for 11 years despite long term rivaroxaban therapy and no further evidence of DVT. Filter removal could have been considered to avoid further complications. With increasing evidence showing that abdominal pain and GI bleeding can be secondary to duodenal penetration of an IVC filter, clinicians should have a high suspicion for this complication which can be confirmed by endoscopy. Based on the success of physician education and programs for patient follow-up for removal, similar ideas should be instituted across systems where there is low IVC filter retrieval to help prevent complications such as migration which is most likely to occur with the passage of time.

\section{Additional Information \\ Disclosures}

Human subjects: All authors have confirmed that this study did not involve human participants or tissue. Conflicts of interest: In compliance with the ICMJE uniform disclosure form, all authors declare the following: Payment/services info: All authors have declared that no financial support was received from any organization for the submitted work. Financial relationships: All authors have declared that they have no financial relationships at present or within the previous three years with any organizations that might have an interest in the submitted work. Other relationships: All authors have declared that there are no other relationships or activities that could appear to have influenced the submitted work.

\section{References}

1. Reddy S, Lakhter V, Zack CJ, et al.: Association between contemporary trends in inferior vena cava filter placement and the 2010 US Food and Drug Administration Advisory. JAMA Intern Med. 2017, 177 :13731374. 10.1001/jamainternmed.2017.2719

2. Moore PS, Andrews JS, Craven TE, et al.: Trends in vena caval interruption. J Vasc Surg. 2010, 52:118-125.e3. 10.1016/i.jvs.2009.09.067

3. Cook AD, Gross BW, Osler TM, et al.: Vena cava filter use in trauma and rates of pulmonary embolism, 2003-2015. JAMA Surg. 2017, 152:724-732. 10.1001/jamasurg.2017.1018

4. PREPIC Study Group: Eight-year follow-up of patients with permanent vena cava filters in the prevention of pulmonary embolism: the PREPIC (Prevention du Risque d'Embolie Pulmonaire par Interruption Cave) randomized study. Circulation. 2005, 112:416-422. 10.1161/CIRCULATIONAHA.104.512834

5. Harvey JJ, Hopkins J, McCafferty IJ, Jones RG: Inferior vena cava filters: what radiologists need to know . Clin Radiol. 2013, 68:721-732. 10.1016/j.crad.2013.01.001

6. Malgor RD, Labropoulos N: A systematic review of symptomatic duodenal perforation by inferior vena cava filters. J Vasc Surg. 2012, 55:856-861. 10.1016/j.jvs.2011.09.082

7. Angel LF, Tapson V, Galgon RE, et al.: Systematic review of the use of retrievable inferior vena cava filters . J Vasc Interv Radiol. 2011, 22:1522-1530.e3. 10.1016/j.jvir.2011.08.024

8. Siracuse JJ, Al Bazroon A, Gill HL, et al.: Risk factors of nonretrieval of retrievable inferior vena cava filters . Ann Vasc Surg. 2015, 29:318-321. 10.1016/j.avsg.2014.08.008

9. Albrecht RM, Garwe T, Carter SM, Maurer AJ: Retrievable inferior vena cava filters in trauma patients: factors that influence removal rate and an argument for institutional protocols. Am J Surg. 2012, 203:297302. 10.1016/j.amjsurg.2011.09.006

10. Stein PD, Alnas M, Skaf E, et al.: Outcome and complications of retrievable inferior vena cava filters . Am J Cardiol. 2004, 94:1090-1093. 10.1016/j.amjcard.2004.06.077

11. Jia Z, Wu A, Tam M, Spain J, Mckinney JM, Wang W: Caval penetration by inferior vena cava filters . Circulation. 2015, 132:944-952. 10.1161/circulationaha.115.016468

12. Deso S, Idakoji i, Kuo W: Evidence-based evaluation of inferior vena cava filter complications based on filter type. Semin Intervent Radiol. 2016, 33:93-100. 10.1055/s-0036-1583208

13. Zhou D, Spain J, Moon E, Mclennan G, Sands MJ, Wang W: Retrospective review of 120 celect inferior vena cava filter retrievals: experience at a single institution. J Vasc Interv Radiol. 2012, 23:1557-1563. 10.1016/j.jvir.2012.08.016

14. Zhu X, Tam MD, Bartholomew J, Newman JS, Sands MJ, Wang W: Retrievability and device-related complications of the G2 filter: a retrospective study of 139 filter retrievals. J Vasc Interv Radiol. 2011, 22:806-812. 10.1016/j.jvir.2011.01.430

15. Caldwell EH, Fridley TL, Erb EL, Fleischer SR: Endovascular retrieval of an inferior vena cava filter with 


\section{Cureus}

simultaneous caval, aortic, and duodenal perforations. Vasc Endovascular Surg. 2012, 46:671-674.

$10.1177 / 1538574412465479$

16. Huang J, Bold M, Rajebi MR: Endovascular retrieval of Greenfield IVC filters 13 and 19 years post placement without major complication. J Radiol Case Rep. 2017, 11:15-25. 10.3941/jrcr.v11i6.3031

17. Duncan C, Trerotola SO, Stavropoulos SW: Endovascular removal of inferior vena cava filters with arterial penetration. J Vasc Interv Radiol. 2018, 29:486-490. 10.1016/j.jvir.2017.12.018 